\title{
Older Men with Bipolar Disorder Diagnosed in Early and Later Life: Physical Health Morbidity and General Hospital Service Use
}

\author{
Osvaldo P. Almeida ${ }^{1,2}$ \\ Graeme J. Hankey ${ }^{1}$ \\ Bu B. Yeap ${ }^{1}$ \\ Jonathan Golledge ${ }^{3}$ \\ Leon Flicker ${ }^{1,2}$ \\ ${ }^{1}$ Medical School, University of Western Australia, Perth, Australia \\ ${ }^{2}$ WA Centre for Health of the University of Western Australia, Perth, Australia \\ ${ }^{3}$ Queensland Research Centre for Peripheral Vascular Disease, College of Medicine and Dentistry, James \\ Cook University, Townsville, Australia
}

Running title: Older Bipolar and General Hospital Use

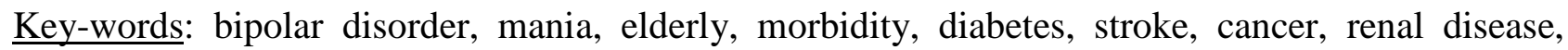
dementia, mortality, health care.

Word count: 3574

\section{Correspondence}

Prof. Osvaldo P. Almeida, Medical School (M577), University of Western Australia, 35 Stirling Highway, Crawley, Perth, WA 6009, Australia. Email: osvaldo.almeida@uwa.edu.au. 


\section{ABSTRACT}

Background: Bipolar disorder (BD) has been associated with greater health morbidity burden, but it is unclear if this association is affected by age at the time of diagnosis and how this might impact on the use of general hospital services.

Methods: Cross-sectional study investigating the prevalence of common medical morbidities among participants with early (EOBD) and late onset diagnosis of BD (LOBD - age at diagnosis $\geq 60$ years) derived from a community-representative sample of 37183 men aged 65-85 years. Cohort study over a follow up period of up to 17.7 years investigating the hazard of general hospital use among older men associated with EOBD and LOBD taking into account age and prevalent medical morbidities.

Results: 250 older men had a recorded diagnosis of BD, 75 of whom had LOBD. Diabetes, stroke and diseases of the respiratory and digestive systems were more frequent in men with than without BD. There were no differences in the distribution of medical morbidities between men with EOBD and LOBD. The adjusted hazard ratio (HR) of contact with general hospital services was significantly higher among men with EOBD $(\mathrm{HR}=1.33$; 95\%CI=1.14, 1.54) and LOBD $(\mathrm{HR}=1.27$, 95\%CI=1.06, 1.51) compared with older men without BD. Older men with EOBD had the highest number of contacts with general hospital services during follow up, although men with EOBD and LOBD did not differ in the number of contacts due to episodes of mania or depression. The medical reasons for contact with general hospital services of men with EOBD and LOBD overlapped but were not identical.

Conclusions: Older men with BD experience greater health morbidity than men without BD. Older men with BD access hospital services for the management of physical morbidities earlier and more frequently than men without BD. Age at the time of diagnosis of BD has limited impact on the risk of contact with general medical services, although subtle differences in the physical morbidity of men with EOBD and LOBD warrant further investigation. 


\section{INTRODUCTION}

Bipolar disorder (BD) is a disabling episodic mental health disorder that is associated with increased morbidity and premature mortality (Crump et al., 2013). This excess morbidity and mortality not only persists but might become more pronounced in old age (Almeida et al., 2016b), raising concerns about the potential impact of $\mathrm{BD}$ on health services as the population ages. The Clinical and Health Outcomes Initiative in Comparative Effectiveness for Bipolar Disorder (Bipolar CHOICE) found that $96 \%$ of 482 participants had at least one other medical comorbidity, with cardiometabolic diseases becoming more prevalent with increasing age (Sylvia et al., 2015). Moreover, the Lithium Treatment - Moderate Dose Use Study (LiTMUS) suggested that concurrent medical morbidity is associated with worse clinical outcomes (Kemp et al., 2014), and the Canadian Community Health Survey reported an increase in the use of health services by these patients (McIntyre et al., 2006).

Older age is the most robust predictor of the use of medical services, with people older than 65 years accounting for nearly half of all hospital bed-days in Australian hospitals, despite representing less than $15 \%$ of the population (AIHW 2018) It is less clear how the presence of BD affects the use of general hospital services in later life, as increasing age could override the effects of BD on medical morbidity. Given the progressive ageing of the World's population, it seems important to understand how mental health disorders, such as BD, might affect physical health and the use of health services as people age. In addition, the Older Adult Task Force of the International Society for Bipolar Disorders has argued that the course of BD might be influenced by the age at the time of onset of symptoms, so that grouping older people into early (EOBD) and late onset (LOBD) may be clinically informative (Sajatovic et al., 2015). For example, BD is associated with increased risk of dementia and this increase in risk seems to be partly driven by the age at the time of onset of BD (Diniz et al., 2017). There is also evidence that EOBD and LOBD may have different clinical 
associations - use of substances (such as alcohol) is associated with EOBD but not LOBD (Almeida et al., 2018b).

We designed this study to investigate the prevalence of common medical morbidities among older men with early and late onset diagnosis of BD. We also sought to determine the risk of contact with general hospital inpatient or emergency services for men with EOBD and LOBD over a follow up period of 17 years, as well as the most frequent medical diagnoses associated with these contacts. We hypothesised that EOBD would be associated with greater medical morbidity than LOBD due to the longer duration of illness, and that the risk and frequency of hospital contacts would be greater in men with EOBD than LOBD.

\section{METHODS}

\section{$\underline{\text { Setting, design and participants }}$}

We used the electoral roll to recruit men aged 65-85 years living in the Perth metropolitan region (registration to vote is compulsory in Australia). Details about the rationale of the study and the steps involved in this process have been reported elsewhere (Almeida et al., 2016a; Norman et al., 2009). This study included only men because the cohort was initially assembled to investigate abdominal aortic aneurysm, which affects predominantly older men (Norman et al., 2009). A total of 38173 eligible men were enrolled between April 1996 and November 1998. They were followed prospectively until they died or until 31 December 2013, whichever occurred first. The Ethics Committees of the University of Western Australia and of the Department of Health of Western Australia approved the study protocol and procedures.

\section{$\underline{\text { Study measures }}$}

We used the Western Australian Data Linkage System (WADLS) to retrieve clinical information about participants' contacts with health services. WADLS brings together information about 
inpatient and outpatient mental health services, hospital morbidity data, emergency departments and death registry (Holman et al., 2008). Clinical diagnoses in WADLS are recorded according to the guidelines of the International Classification of Diseases (ICD): ICD-8 from $1^{\text {st }}$ January 1966 to $31^{\text {st }}$ December 1969, ICD-9 from $1^{\text {st }}$ January 1970 to $30^{\text {th }}$ June 1999 , and ICD-10 from the $1^{\text {st }}$ July 1999. WADLS also records the start and end dates of all health events. The death registry provided the date of death of the participants who died during follow up.

We used the following ICD codes to establish the diagnosis of bipolar disorder (BD) among participants: 296.1 and 296.3 (ICD-8), 296.0, 296.1, 296.4, 296.5, 296.6, 296.7, 296.80 and 296.81 (ICD-9), and F30 or F31 (ICD-10). We considered that the date of onset of BD was the same as the date of the first ever contact with the diagnosis of BD. If the diagnosis of a depressive disorder occurred before the diagnosis of BD was established, then the date of contact with a depressive disorder was considered the date of onset of BD. The ICD codes that identified depressive episodes were: 296.0 and 300.4 (ICD-8), 296.2, 296.3, 311 and 300.4 (ICD-9), and F32, F33, F34.1 and F38.10 (ICD-10). As the earliest mental health records available were dated $1^{\text {st }}$ January 1966 , the minimum age of onset recordable for our cohort would have been 35 years (i.e., 30 years before the start of the study). Based on existing recommendations (Sajatovic et al., 2015), we used age 60 years or older to define BD with late onset (LOBD), so that all participants with onset of BD before age 60 years were grouped under the rubric 'early onset BD' (EOBD). Of note, the diagnoses of schizophrenia and delusional disorder (ICD codes 295, 297, F20, F22, F23, F25, F28 and F29) had precedence over the diagnosis of $\mathrm{BD}$.

We calculated the age of participants (in years) by subtracting the date of birth from the date of enrolment into the study, and retrieved data on diabetes, hypertension, ischaemic heart disease, stroke, cancers (except skin cancer), chronic respiratory diseases, gastrointestinal and renal diseases and dementia using the following ICD codes recorded in WADLS: 
- diabetes ICD-8 and 9 codes 249 and 250, and ICD-10 codes E08-13;

- $\quad$ hypertension ICD-8 and 9 codes 401-403 and ICD-10 codes I10 and I12;

- ischaemic heart disease ICD-8 and 9 codes 390-398, 402, 404, 410-429, and ICD-10 codes I0009, I11, I13, I20-29, I50-51;

- $\quad$ stroke ICD-8 and 9 codes 430-434, 436-438, and ICD-10 codes I60-69;

- cancers ICD-8 and ICD-9 codes 140-209, and ICD-10 codes C00-C97;

- respiratory diseases ICD-8 and ICD-9 codes 490-496 and 507-519, and ICD-10 codes J00-09, J20-39, J40-47 and J60-99;

- gastrointestinal diseases ICD-8 and ICD-9 codes 520-537, 540-543, 5550-553, 555-589, and ICD-10 codes K00-K99;

- renal diseases ICD-8 and ICD-9 codes 580-589, and ICD-10 codes N00-07, N17-19 and N2527;

- $\quad$ dementia ICD-8 code 290; ICD-9 codes 290, 294.1, 294.2, 331.0, 331.1, 331.2, 331.82; ICD-10 codes F00-F03, G30, G31.0, G31.1, G31.83.

\section{$\underline{\text { Statistical analyses }}$}

We used the statistical software Stata 15.1 to manage and analyse the data (StataCorp LLC, Revision 8 May 2018). Descriptive statistics summarised categorical variables as counts and proportions (\%), continuous variables as mean, range, and standard deviation of the mean (SD), and ordinal data as median and inter-quartile range (IQR). We used one-way analysis of variance to compare the ages of participants according to clinical group: no bipolar, bipolar with illness onset before and at or after age 60 years, as per advice of the Older Adult Task Force of the International Society for Bipolar Disorders (Sajatovic et al., 2015). Pearson chi-square statistic $\left(X^{2}\right)$ was applied to examine differences in the cross-sectional distribution of health morbidities between men without BD and those with EOBD and LOBD. Similarly, Kruskal-Wallis non-parametric analysis of variance $\left(X^{2}\right)$ was used to investigate between group differences in the number of contacts with 
health services during the follow up period. These analyses were followed by between groupcomparisons using Mann-Whitney non-parametric test. Finally, we used Cox regression (Breslow method) to determine the hazard ratio (HR) of contact with general hospital services during the follow up period. In this model, we split and joined time-span sets according to the diagnosis of BD (including the age of onset), so that men without BD contributed data as controls until the time of diagnosis and as cases thereafter. We used age as the time scale in the Cox regression models in order to control as accurately as possible the effect of age on the risk of health contacts (Breslow et al., 1983). Similarly, we used Cox regression to determine the risk of health contacts associated with specific health disorders over time for men with EOBD and LOBD relative to men without BD. Alpha was set at 5\% and all risk estimates were reported alongside their respective $95 \%$ confidence interval (95\%CI).

\section{RESULTS}

The age of the 38173 participants ranged from 65.0 to 85.7 years. The sample included 250 men with $\mathrm{BD}$, of whom 75 had the diagnosis recorded for the first ever time at or after age 60 years (LOBD). One-way analysis of variance showed that men with EOBD were, on average, 1.3 years younger than men without BD, whereas men with LOBD were 1.9 and 3.3 years older than men without $\mathrm{BD}$ and those with EOBD respectively $(\mathrm{F}=13.83, \mathrm{df}=2, \mathrm{p}<0.001)$. Table 1 summarises the demographic and clinical characteristics of participants. Men with BD showed a higher prevalence of diabetes, stroke and diseases of the respiratory and digestive systems, but differences between participants with EOBD and LOBD were negligible ( $>0.470$ for all comparisons). We also investigated the association between cardiovascular risk factors (diabetes and hypertension) and diseases (ischaemic heart disease and stroke) with BD: both EOBD (52.6\%; OR=1.50, 95\%CI=1.11, 2.03) and LOBD (62.7\%; OR=1.86, 95\%CI=1.16, 2.99) were associated with greater age-adjusted odds of cardiovascular conditions compared with no BD (44.6\%). 


\section{TABLE 1}

We followed participants for $11.2 \pm 5.3$ years (range: 0.0 to 17.7 years). Over this period, 36675 (96.1\%) had contact with general hospital services at least once, and 25701 (67.3\%) had at least one contact with emergency departments. The risk of hospital contact (including emergency) was higher among men with EOBD and LOBD than men without BD, but there was no risk-difference between men with EOBD and LOBD ( $\mathrm{p}=0.693$ ) (Figure 1). The median number of hospital contacts during the follow up period was 11 (IRQ=5 to 19 ), 14 (IQR=7 to 26) and 10 (IQR=6 to 19) for men without BD and for those with EOBD and LOBD respectively (Kruskal-Wallis $X^{2}=10.02$, df=2, $\mathrm{p}=0.007)$. Men with EOBD had a higher frequency of hospital contacts than men without BD ( $p=0.002)$. The number of hospital contacts for men with LOBD did not differ from men without $\operatorname{BD}(p=0.894)$ or men with EOBD $(p=0.067)$.

\section{FIGURE 1}

Table 2 shows the most frequent clinical diagnoses associated with health contacts during follow up. Men with LOBD had higher risk of health contact associated with stroke than men without BD, whereas men with EOBD had greater risk of contacts associated with the diagnosis of cancer and diseases of the respiratory system. The risk of renal diseases, dementia and death were higher among older men with both EOBD and LOBD compared with men without BD. Older men with EOBD and LOBD did not differ in the distribution of any of the clinical diagnoses listed during the follow up period ( $\mathrm{p}>0.05$ for all comparisons).

\section{TABLE 2}


The number of contacts with mental health services with distinctive episodes (i.e., not due to ongoing care) ranged from 0 (zero) to 8. Kruskal-Wallis non-parametric analysis of variance (adjusted for ties) showed that the groups differed (Kruskal-Wallis $X^{2}=118.10, \mathrm{df}=2, \mathrm{p}<0.001$ ), with subsequent analyses showing that this was due to differences between men without BD and both EOBD $(\mathrm{p}<0.001)$ and LOBD ( $\mathrm{p}=0.001)$. Participants with EOBD and LOBD had a similar number of mental health contacts during follow up $(\mathrm{p}=0.187)$. Three men with EOBD had a manic episode during follow up, 3 had 2, 1 had 3, and 1 had 8. Two of these men also had 1 depressive episode, 2 had 2, and 1 had 3 depressive episodes during follow up. Among participants with LOBD, 2 experienced 1 new episode of mania during follow up and 1 had 2 new depressive episodes. There were no statistically significant differences in the number of manic and depressive episodes according to age at first contact $(\mathrm{p}>0.05)$.

\section{DISCUSSION}

The results of this study showed that older adults with BD have higher prevalence of medical morbidities such as diabetes, stroke and renal and gastrointestinal diseases, although age at the time of first contact with a diagnosis of BD (i.e., EOBD vs LOBD) did not influence their frequency distribution. The adjusted risk of contact with general hospital services during the 17-year follow up was about $30 \%$ higher for older men with than without BD and this risk was not affected by age at the time of first contact with BD. However, men with LOBD had higher risk of health contacts associated with the diagnosis of stroke than men without $\mathrm{BD}$, whereas older participants with EOBD had higher risk of health contacts associated with the diagnosis of cancer and respiratory diseases. Both men with EOBD and LOBD had increased risk of general hospital contacts associated with the diagnosis of renal diseases and dementia; they were also more likely to die during follow up than men without BD. Older men with EOBD had the highest number of contacts with general hospital services during follow up, although men with EOBD and LOBD did not differ in the number of contacts due to episodes of mania or depression. Taken together, these findings 
suggest that there is a higher prevalence of medical morbidities among older men with $\mathrm{BD}$, regardless the age at the time of diagnosis. They also show that older men with $\mathrm{BD}$, compared to those without, are greater users of general hospital services and that the frequency of contact may be particularly high among those with EOBD.

\section{$\underline{\text { Strengths and limitations }}$}

This study recruited a large community-representative sample of older men, which allowed us to identify a sufficiently large number of people with BD. By using WADLS we were able to monitor the health movements of participants and to circumvent concerns about loss to follow up (the migratory movements of older Western Australians are negligible) (ABS 2015). In addition, the extended follow up period meant that nearly most participants had the opportunity of accruing a health contact in WADLS, and our approach to the analysis of the data made our findings more robust by taking into account the contribution of incident cases of BD.

The diagnosis of BD retrieved from WADLS was based on health administrative data rather than a structured clinical assessment. Nonetheless, our hierarchical approach to diagnosis, which would have decreased the risk of misclassification, yielded a prevalence estimate similar to those of studies using different strategies to identify cases (Depp et al., 2005; Merikangas et al., 2011). We acknowledge, however, that our definition of EOBD and LOBD was based on age at the time of first contact with the health services. It is possible that early mild affective episodes could have been missed and that some misclassification could have ensued. This would have affected primarily the group with LOBD (because early mild affective episodes might not have been recorded in WADLS). Such a bias would have diluted the differences between EOBD and LOBD. In a previous study we have shown that changing the age that defines LOBD has limited effect on clinical associations, which suggests that error associated with the definition of EOBD and LOBD was most likely minimal (Almeida et al., 2018a; Almeida et al., 2016b). We are also mindful of the fact that 
WADLS records are only available from 1966, which means that earlier mental health contacts would have not been available. Hence, the earliest possible age of onset of symptoms in our cohort would have been 35 years. However, it seems improbable that this would have introduced error in our classification of EOBD and LOBD, as one would expect nearly all people with onset of BD symptoms in their teens or early 20s to have experienced further affective episodes before reaching 60 years of age (Berk et al., 2007b; Coryell et al., 2013). Moreover, as we did not have access to data on treatment and could not confidently calculate the number of episodes that participants had experienced, we were unable to investigate the contribution of these factors to the outcomes of this study. Limited power (due to the relatively small number of men with BD, particularly LOBD) and random error cannot be dismissed entirely in an observational study such as this, and we also accept that our findings are limited to men and cannot be directly extended to women, although data from other sources indicate a similar burden of physical morbidity for women with BD (Carney and Jones, 2006).

\section{Interpretation}

Physical health morbidities such as cardiovascular diseases and diseases of the respiratory and digestive systems are more prevalent among older men with than without $\mathrm{BD}$, although the age at first contact with health services (i.e., EOBD vs LOBD) does not seem to affect the distribution of these conditions. We had anticipated that older men with EOBD would experience greater physical health burden than participants with LOBD because of deteriorating lifestyle associated with BD as well as the long term implications of using mood stabilisers (Almeida et al., 2018b; Ng et al., 2009; Shine et al., 2015; Vancampfort et al., 2016). There is evidence that people with BD display disrupted lifestyle patterns during euthymic states (Robb et al., 1997), and it is possible that these lifestyle disruptions may occur even before the onset of clinical symptoms (Berk et al., 2007a; Skjelstad et al., 2010). In that case, some of the behaviours commonly associated with BD (e.g.. high body mass index, substance misuse, physical inactivity) could precede the overt manifestation 
of BD (Marangoni et al., 2016). These behavioural patterns could, in turn, contribute to increase the physical morbidity of people with BD as well as of those at risk. Furthermore, mortality data from the Swedish National Cohort Study suggest that the use of lithium, valproate and atypical antipsychotics have negligible effect on the risk of death of people with BD (Crump et al., 2013), which would be consistent with the interpretation that common medications used in the treatment of BD do not add substantial health burden (and health contacts) to patients, thereby making the physical morbidity difference between EOBD and LOBD negligible. It is also possible that differences in hospital contact between EOBD and LOBD become weaker as the population ages and the number of competing medical morbidities from various causes increases (McCaul et al., 2015). The use of age $\geq 60$ years to define LOBD did not seem able to discriminate between those who would or not develop dementia during follow up, although in at least some cases manic or hypomanic symptoms may represent an early clinical expression of dementia (Almeida et al., 2016b) or may indicate a common vulnerability to conditions such as frontotemporal dementia (Cerami et al., 2011; Floris et al., 2013). Our findings suggest overlapping, albeit not identical, medical reasons for contact with general hospital services for older men with EOBD and LOBD, and show an excess of morbidity across most health systems. Others had already reported negligible differences between older adults with BD who had onset of symptoms before or after age 40 years (Chu et al., 2010), although subsequent critical appraisals had suggested that a later age at onset (e.g., $\geq 60$ years) could be informative in terms of clinical outcomes (Sajatovic et al., 2015).

The number of older men with BD who experienced additional affective episodes during follow up was small, and of those most were due to mania. This suggests that the recurrence of manic symptoms may become less frequent as people age. A Danish study comparing old and young adults with BD found that people with onset of BD after age 50 years had more frequent and severe depressive episodes, while younger adults had more contacts associated with severe manic episodes (Kessing, 2006). Our findings confirm that the recurrence of manic episodes may become less 
frequent in later life but failed to show evidence of increased contacts associated with depression. This could have been due to the fact that some episodes of depression experienced by these men were diagnosed and managed in primary care, which would have made them inaccessible to WADLS (Almeida et al., 2015). If that is the case, then those depressive episodes would have been relatively mild, as severe depression invariably comes to the attention of mental health services in Western Australia (and are then recorded in WADLS). Taken together, these results suggest that the course of affective symptoms seems to be relatively benign for most men with BD who reach old age. We are also mindful of the fact that differential mortality could conceivably confound the association between BD with hospital contacts, although that seems an unlikely explanation for our findings because older men with EOBD and LOBD have similar mortality rates during follow up (Almeida et al., 2018a).

In summary, our findings showed that older men with BD experience greater health morbidity than men without BD. Older men with $\mathrm{BD}$ access hospital services for the management of physical morbidities earlier and more frequently than men without BD and, not surprisingly, die earlier. Age at the time of diagnosis of BD has limited impact on the risk of contact with general medical services, although subtle differences in the physical morbidity of men with EOBD and LOBD warrant further investigation. 


\section{REFERENCES}

ABS 2015. Australian Bureau of Statistics. 3412.0 - Migration, Australia, 2013-14. ABS, Canberra: Australia.

AIHW 2018. Australian Institute of Health and Welfare 2018. Admitted patient care 2016-17: Australian hospital statistics. Health services series no. 84. Cat. no. HSE 201. AIHW, Canberra.

Almeida, O.P., Hankey, G.J., Yeap, B.B., Golledge, J., Flicker, L., 2015. Prevalence, associated factors, mood and cognitive outcomes of traumatic brain injury in later life: the health in men study (HIMS). Int J Geriatr Psychiatry 30, 1215-1223.

Almeida, O.P., Hankey, G.J., Yeap, B.B., Golledge, J., Flicker, L., 2018a. Older men with bipolar disorder: Clinical associations with early and late onset illness. Int J Geriatr Psychiatry.

Almeida, O.P., Hankey, G.J., Yeap, B.B., Golledge, J., Flicker, L., 2018b. Substance use among older adults with bipolar disorder varies according to age at first treatment contact. J Affect Disord 239, 269-273.

Almeida, O.P., McCaul, K., Hankey, G.J., Yeap, B.B., Golledge, J., Flicker, L., 2016a. Affective Disorders, Psychosis and Dementia in a Community Sample of Older Men with and without Parkinson's Disease. PLoS One 11, e0163781.

Almeida, O.P., McCaul, K., Hankey, G.J., Yeap, B.B., Golledge, J., Flicker, L., 2016b. Risk of dementia and death in community-dwelling older men with bipolar disorder. Br J Psychiatry 209, 121-126.

Berk, M., Conus, P., Lucas, N., Hallam, K., Malhi, G.S., Dodd, S., Yatham, L.N., Yung, A., McGorry, P., 2007a. Setting the stage: from prodrome to treatment resistance in bipolar disorder. Bipolar Disord 9, 671-678.

Berk, M., Dodd, S., Callaly, P., Berk, L., Fitzgerald, P., de Castella, A.R., Filia, S., Filia, K., Tahtalian, S., Biffin, F., Kelin, K., Smith, M., Montgomery, W., Kulkarni, J., 2007b. History of illness prior to a diagnosis of bipolar disorder or schizoaffective disorder. J Affect Disord 103, 181186.

Breslow, N.E., Lubin, J.H., Marek, P., Langholz, B., 1983. Multiplicative models and cohort analysis. J Am Stat Assoc 78, 1-12.

Carney, C.P., Jones, L.E., 2006. Medical comorbidity in women and men with bipolar disorders: a population-based controlled study. Psychosom Med 68, 684-691.

Cerami, C., Marcone, A., Galimberti, D., Villa, C., Scarpini, E., Cappa, S.F., 2011. From genotype to phenotype: two cases of genetic frontotemporal lobar degeneration with premorbid bipolar disorder. J Alzheimers Dis 27, 791-797.

Chu, D., Gildengers, A.G., Houck, P.R., Anderson, S.J., Mulsant, B.H., Reynolds, C.F., 3rd, Kupfer, D.J., 2010. Does age at onset have clinical significance in older adults with bipolar disorder? Int J Geriatr Psychiatry 25, 1266-1271.

Coryell, W., Fiedorowicz, J., Leon, A.C., Endicott, J., Keller, M.B., 2013. Age of onset and the prospectively observed course of illness in bipolar disorder. J Affect Disord 146, 34-38. 
Crump, C., Sundquist, K., Winkleby, M.A., Sundquist, J., 2013. Comorbidities and mortality in bipolar disorder: a Swedish national cohort study. JAMA Psychiatry 70, 931-939.

Depp, C.A., Lindamer, L.A., Folsom, D.P., Gilmer, T., Hough, R.L., Garcia, P., Jeste, D.V., 2005. Differences in clinical features and mental health service use in bipolar disorder across the lifespan. Am J Geriatr Psychiatry 13, 290-298.

Diniz, B.S., Teixeira, A.L., Cao, F., Gildengers, A., Soares, J.C., Butters, M.A., Reynolds, C.F., 3rd, 2017. History of Bipolar Disorder and the Risk of Dementia: A Systematic Review and MetaAnalysis. Am J Geriatr Psychiatry 25, 357-362.

Floris, G., Borghero, G., Cannas, A., Stefano, F.D., Murru, M.R., Corongiu, D., Cuccu, S., Tranquilli, S., Marrosu, M.G., Chio, A., Marrosu, F., 2013. Bipolar affective disorder preceding frontotemporal dementia in a patient with C9ORF72 mutation: is there a genetic link between these two disorders? J Neurol 260, 1155-1157.

Holman, C.D., Bass, A.J., Rosman, D.L., Smith, M.B., Semmens, J.B., Glasson, E.J., Brook, E.L., Trutwein, B., Rouse, I.L., Watson, C.R., de Klerk, N.H., Stanley, F.J., 2008. A decade of data linkage in Western Australia: strategic design, applications and benefits of the WA data linkage system. Aust Health Rev 32, 766-777.

Kemp, D.E., Sylvia, L.G., Calabrese, J.R., Nierenberg, A.A., Thase, M.E., Reilly-Harrington, N.A., Ostacher, M.J., Leon, A.C., Ketter, T.A., Friedman, E.S., Bowden, C.L., Rabideau, D.J., Pencina, M., Iosifescu, D.V., Li, T.S.G., 2014. General medical burden in bipolar disorder: findings from the LiTMUS comparative effectiveness trial. Acta Psychiatr Scand 129, 24-34.

Kessing, L.V., 2006. Diagnostic subtypes of bipolar disorder in older versus younger adults. Bipolar Disord 8, 56-64.

Marangoni, C., Hernandez, M., Faedda, G.L., 2016. The role of environmental exposures as risk factors for bipolar disorder: A systematic review of longitudinal studies. J Affect Disord 193, 165174.

McCaul, K.A., Almeida, O.P., Norman, P.E., Yeap, B.B., Hankey, G.J., Golledge, J., Flicker, L., 2015. How many older people are frail? Using multiple imputation to investigate frailty in the population. J Am Med Dir Assoc 16, 439 e431-437.

McIntyre, R.S., Konarski, J.Z., Soczynska, J.K., Wilkins, K., Panjwani, G., Bouffard, B., Bottas, A., Kennedy, S.H., 2006. Medical comorbidity in bipolar disorder: implications for functional outcomes and health service utilization. Psychiatr Serv 57, 1140-1144.

Merikangas, K.R., Jin, R., He, J.P., Kessler, R.C., Lee, S., Sampson, N.A., Viana, M.C., Andrade, L.H., Hu, C., Karam, E.G., Ladea, M., Medina-Mora, M.E., Ono, Y., Posada-Villa, J., Sagar, R., Wells, J.E., Zarkov, Z., 2011. Prevalence and correlates of bipolar spectrum disorder in the world mental health survey initiative. Arch Gen Psychiatry 68, 241-251.

Ng, F., Mammen, O.K., Wilting, I., Sachs, G.S., Ferrier, I.N., Cassidy, F., Beaulieu, S., Yatham, L.N., Berk, M., International Society for Bipolar, D., 2009. The International Society for Bipolar Disorders (ISBD) consensus guidelines for the safety monitoring of bipolar disorder treatments. Bipolar Disord 11, 559-595. 
Norman, P.E., Flicker, L., Almeida, O.P., Hankey, G.J., Hyde, Z., Jamrozik, K., 2009. Cohort Profile: The Health In Men Study (HIMS). Int J Epidemiol 38, 48-52.

Robb, J.C., Cooke, R.G., Devins, G.M., Young, L.T., Joffe, R.T., 1997. Quality of life and lifestyle disruption in euthymic bipolar disorder. J Psychiatr Res 31, 509-517.

Sajatovic, M., Strejilevich, S.A., Gildengers, A.G., Dols, A., Al Jurdi, R.K., Forester, B.P., Kessing, L.V., Beyer, J., Manes, F., Rej, S., Rosa, A.R., Schouws, S.N., Tsai, S.Y., Young, R.C., Shulman, K.I., 2015. A report on older-age bipolar disorder from the International Society for Bipolar Disorders Task Force. Bipolar Disord 17, 689-704.

Shine, B., McKnight, R.F., Leaver, L., Geddes, J.R., 2015. Long-term effects of lithium on renal, thyroid, and parathyroid function: a retrospective analysis of laboratory data. Lancet 386, 461-468.

Skjelstad, D.V., Malt, U.F., Holte, A., 2010. Symptoms and signs of the initial prodrome of bipolar disorder: a systematic review. J Affect Disord 126, 1-13.

Sylvia, L.G., Shelton, R.C., Kemp, D.E., Bernstein, E.E., Friedman, E.S., Brody, B.D., McElroy, S.L., Singh, V., Tohen, M., Bowden, C.L., Ketter, T.A., Deckersbach, T., Thase, M.E., ReillyHarrington, N.A., Nierenberg, A.A., Rabideau, D.J., Kinrys, G., Kocsis, J.H., Bobo, W.V., Kamali, M., McInnis, M.G., Calabrese, J.R., 2015. Medical burden in bipolar disorder: findings from the Clinical and Health Outcomes Initiative in Comparative Effectiveness for Bipolar Disorder study (Bipolar CHOICE). Bipolar Disord 17, 212-223.

Vancampfort, D., Firth, J., Schuch, F., Rosenbaum, S., De Hert, M., Mugisha, J., Probst, M., Stubbs, B., 2016. Physical activity and sedentary behavior in people with bipolar disorder: A systematic review and meta-analysis. J Affect Disord 201, 145-152. 
Table 1. Demographic and clinical characteristics of participants according to the presence of bipolar disorder and age at onset.

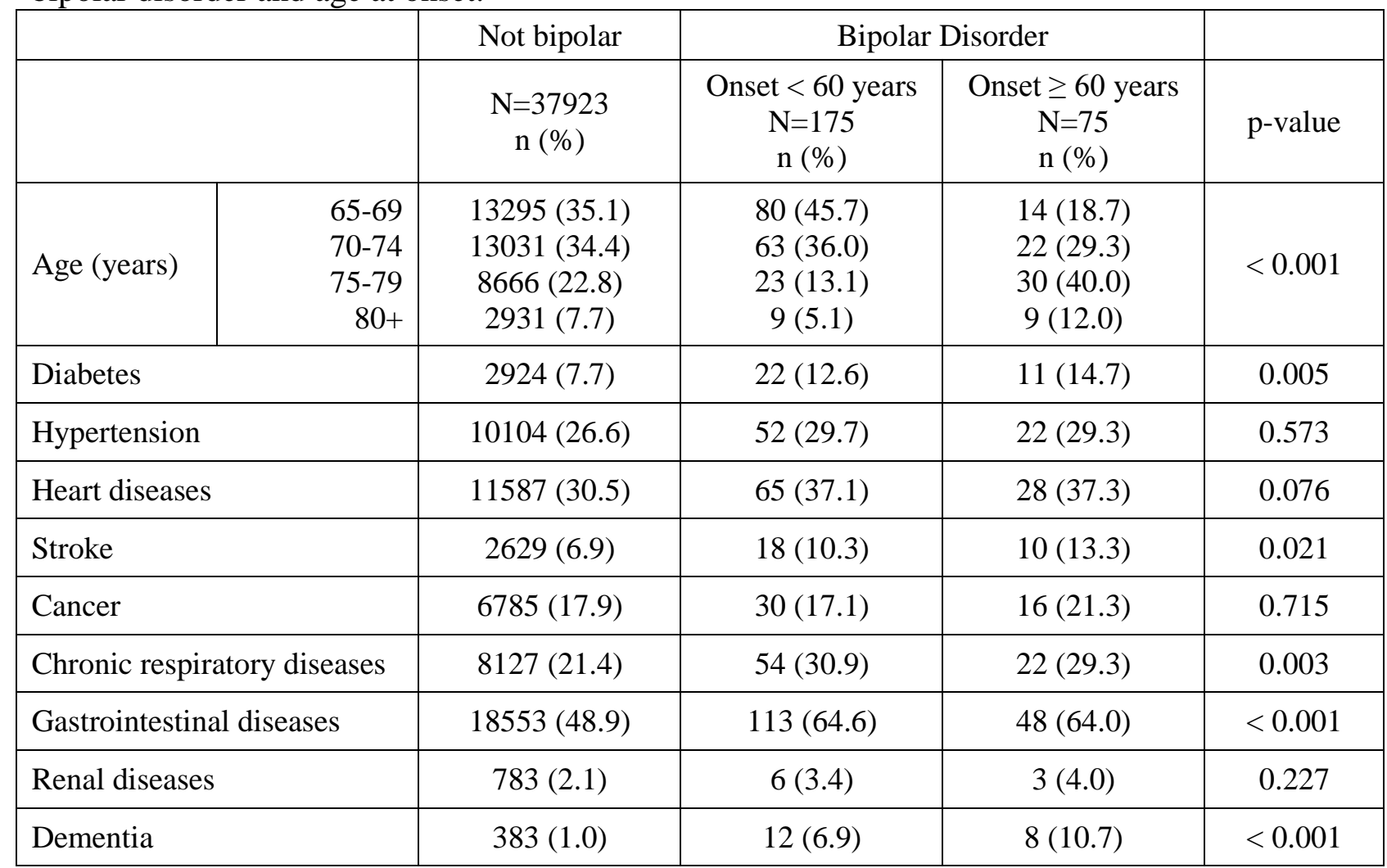


Table 2. Risk of health events during the follow up period of up to 17.7 years among older men with bipolar disorder relative to those without.

\begin{tabular}{|l|c|c|}
\hline & \multicolumn{2}{|c|}{ Bipolar Disorder } \\
\hline Ischaemic heart disease & $\begin{array}{c}\text { Onset }<60 \text { years } \\
\text { HR }(95 \% \mathrm{CI})\end{array}$ & $\begin{array}{c}\text { Onset } \geq 60 \text { years } \\
\text { HR }(95 \% \mathrm{CI}) *\end{array}$ \\
\hline Stroke & $1.22(0.96,1.56)$ & $0.99(0.68,1.44)$ \\
\hline Cancer & $1.32(0.95,1.84)$ & $\mathbf{1 . 7 4}(\mathbf{1 . 1 2}, \mathbf{2 . 6 9})$ \\
\hline Chronic respiratory diseases & $\mathbf{1 . 4 0}(\mathbf{1 . 1 3}, \mathbf{1 . 7 2})$ & $0.94(0.65,1.37)$ \\
\hline Digestive diseases & $\mathbf{1 . 6 1}(\mathbf{1 . 2 7}, \mathbf{2 . 0 3})$ & $1.15(0.79,1.67)$ \\
\hline Renal diseases & $1.22(0.89,1.67)$ & $1.46(0.95,2.24)$ \\
\hline Dementia & $\mathbf{1 . 4 6}(\mathbf{1 . 2 0}, \mathbf{1 . 7 8})$ & $\mathbf{1 . 6 0}(\mathbf{1 . 2 0 ,} \mathbf{2 . 1 3})$ \\
\hline Death & $\mathbf{2 . 5 8 ( 1 . 9 8 , 3 . 3 6 )}$ & $\mathbf{2 . 4 9}(\mathbf{1 . 6 8}, \mathbf{3 . 6 9})$ \\
\hline
\end{tabular}

*HR (95\%CI): hazard ratio and respective 95\% confidence interval derived from a Cox regression model that took into account age and prevalent cases of the relevant health outcome (except for death). 


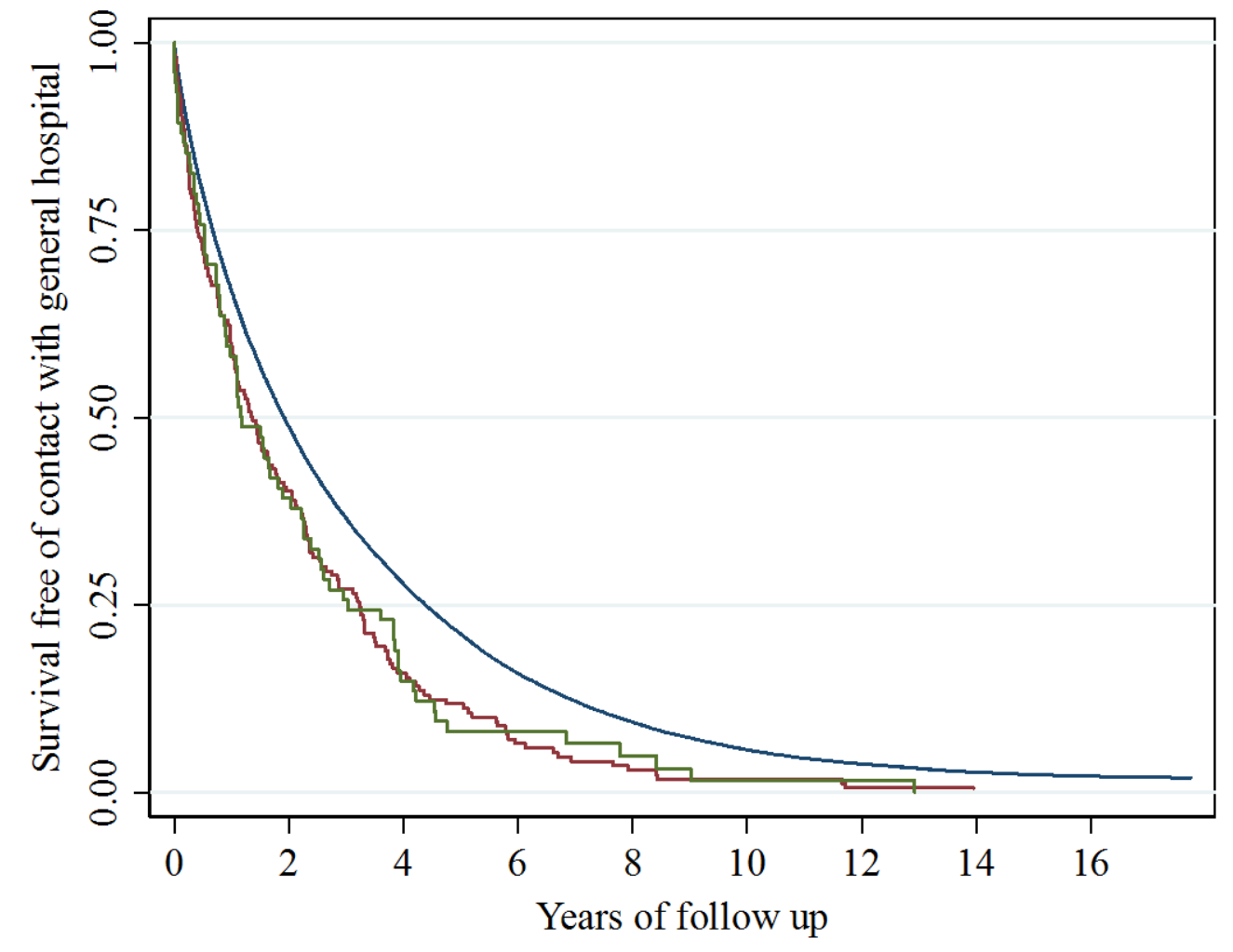

\begin{tabular}{|c|c|c|c|c|c|c|c|c|c|}
\hline $\begin{array}{l}\text { Number at risk: } \\
\text { Not bipolar (navy line) }\end{array}$ & 37923 & 18217 & 10261 & 5800 & 3411 & 2068 & 1355 & 968 & 518 \\
\hline Bipolar contact $<60$ ye ars (red line) & 175 & 68 & 27 & 11 & 5 & 3 & 1 & 0 & 0 \\
\hline Bipolar contact $\geq 60$ years (green line) & 75 & 29 & 11 & 5 & 3 & 1 & 1 & 0 & 0 \\
\hline
\end{tabular}

Figure 1. Proportion of older men who had no contact with general hospital services over the follow-up period of up to 17.7 years. The navy line depicts men without bipolar disorder (BD). The red and green lines depict men with $\mathrm{BD}$ with early $(<60$ years) and late onset $(\geq 60$ years) respectively. The hazard ratio of contact with general hospital services relative to men without BD was $1.33(95 \% \mathrm{CI}=1.14,1.54)$ and $1.27(95 \% \mathrm{CI}=1.06,1.51)$ for men with first ever contact with the diagnosis of $\mathrm{BD}<$ and $\geq 60$ years respectively. These analyses were adjusted for age and for the presence of prevalent diabetes and diseases of the heart, stroke, and respiratory and digestive systems. Incident cases of late onset BD acted as controls until the date of the diagnosis and as cases thereafter. 